Research Article

\title{
Filippov Ratio-Dependent Prey-Predator Model with Threshold Policy Control
}

\author{
Xianghong Zhang and Sanyi Tang \\ College of Mathematics and Information Science, Shaanxi Normal University, Xian, Shaanxi 710062, China \\ Correspondence should be addressed to Sanyi Tang; sytang@snnu.edu.cn
}

Received 10 June 2013; Revised 23 August 2013; Accepted 2 September 2013

Academic Editor: Hamid Reza Karimi

Copyright ( 12013 X. Zhang and S. Tang. This is an open access article distributed under the Creative Commons Attribution License, which permits unrestricted use, distribution, and reproduction in any medium, provided the original work is properly cited.

The Filippov ratio-dependent prey-predator model with economic threshold is proposed and studied. In particular, the sliding mode domain, sliding mode dynamics, and the existence of four types of equilibria and tangent points are investigated firstly. Further, the stability of pseudoequilibrium is addressed by using theoretical and numerical methods, and also the local sliding bifurcations including regular/virtual equilibrium bifurcations and boundary node bifurcations are studied. Finally, some global sliding bifurcations are addressed numerically. The globally stable touching cycle indicates that the density of pest population can be successfully maintained below the economic threshold level by designing suitable threshold policy strategies.

\section{Introduction}

Ordinary differential equation models (ODE models) are widely used to describe the dynamics between predators and their prey, which has long been and will continue to be one of significant fields in mathematical ecology owing to its universal existence and importance $[1,2]$. The simplest preypredator dynamic model is the Lotka-Volterra model [3], which has been modified in many ways since its original and realism formulation in the 1920s.

One important component of the prey-predator relation is predator's functional response which refers to the change in the density of prey attached per unit time per predator as the prey density changes and makes the prey-predator system more realistic. There are several famous functional response types in previous work, which are monotonically increasing and uniformly bounded functions in the first quadrant. Another functional response is the MichaelisMenten (or Holling-type II) functional response, which is the most common type of functional response among arthropod predators $[4,5]$. It takes the form $p(x, y)=a x /(b+x)$, where $a$ and $b$ are positive constants that stand for capturing rate, and half capturing saturation constant, respectively.

Considering predators having to search for food, a more suitable general prey-predator theory based on the so-called ratio-dependent theory is involved. It can be roughly comprehended as the per capita predator growth rate should be a function of the ratio of prey to predator abundance. And it is also strongly supported by numerous fields, laboratory experiments, and observations [6-8].

Therefore, we can write the ratio-dependent prey-predator model with Michaelis-Menten functional response as follows:

$$
\begin{gathered}
\dot{x}(t)=r x\left(1-\frac{x}{k}\right)-\frac{a x y}{b y+x}, \\
\dot{y}(t)=-\delta y+\frac{\beta a x y}{b y+x},
\end{gathered}
$$

where $x$ and $y$ represent the density of prey (pest) and predator (natural enemy), respectively. The prey is assumed to grow logistically and $k$ is the carrying capacity of prey. The positive constants $r$ and $\delta$ stand for intrinsic growth rate of prey and mortality rate of predator, respectively. $\beta$ denotes the conversion rate of prey captured by predator.

In population, both ecologist and mathematicians are interested in the ratio-dependent prey-predator model with Michaelis-Menten functional response [2, 7-9]. Hsu et al. [7] resolved a complete classification of the asymptotic behavior of the solutions of ratio-dependent model with the MichaelisMenten functional response. They also studied the global 
stability of all equilibria in various cases and reconsidered the uniqueness of limit cycle.

It is well known that pests have been one of the principal threats to crops, important plants, animals, and humans all over the world. Therefore, it is necessary to apply acceptable and effective strategies to control pest outbreak. In practice, it is impossible to eradicate the pests completely, nor is it biologically or economically desirable. Integrated pest management (IPM) is a long term management strategy [10-12], which uses a combination of biological, cultural, and chemical tactics so as to lower cost to the growers, minimize effect on the environment, and maintain pest population below the economic injury level (EIL). On the basis of IPM, biological strategy is useful and effective to suppress pest population, such as releasing beneficial natural enemies; culture strategy makes the environment less favorable to pests, such as catching or harvesting artificially. In most cropping systems, when the above two tactics are unable to keep pest population below the ET, chemical strategy (i.e., insecticide) is still a principal means to control pests and prevent economic loss. Thus, in order to control pest outbreak, we should carry out control strategies when the number of pests reaches or exceeds the ET which is lower than the EIL, and the control strategies should be suspended once the density of pest population falls below the ET, which is the so-called threshold policy control (TPC). Considering IPM strategies, either fixed moment or state-dependent impulsive models with the ratio-dependent or Michaelis-Menten-type response function of prey-predator model have been studied in [13-16].

However, Zhao et al. [17] have stated some disadvantages of the impulsive differential equation models mentioned above. First, in the fixed moment impulsive model, without consideration whether the density of pest reaches the ET or not, control strategies are invariably implemented, which leads to consumption of vast resources. Second, in reality, all kinds of control strategies need some time and cannot be finished instantaneously, but in the state-dependent impulsive differential models, control strategies are carried out instantaneously, which is not reasonable.

Therefore, we use Filippov system which is a vector differential equation with discontinuous right-hand side to describe prey-predator model with both noninstantaneous interventions and the threshold policy. Recently, although Filippov systems have been widely utilized in science and engineering, including harvesting thresholds, oil well drilling, and liquid-gas reaction [18-23]. However, very little is involved that they are used to investigate the ratio-dependent-type predator-prey model with MichaelisMenten-type functional response. We assume that a proportion of preys are caught or transferred (culture strategy) or killed (chemical strategy), denoted by $q_{1}$; a proportion of predators are released (biological strategy), denoted by $q_{2}$. So we have the following control model for $x>$ ET:

$$
\begin{gathered}
\dot{x}(t)=r x\left(1-\frac{x}{k}\right)-\frac{a x y}{b y+x}-q_{1} x, \\
\dot{y}(t)=-\delta y+\frac{\beta a x y}{b y+x}+q_{2} y .
\end{gathered}
$$

In this paper, we aim to give a detailed analysis of Filippov ratio-dependent prey-predator model with threshold policy control, which describes that control measures are implemented only when the density of pest in a population exceeds the ET. We investigate the sliding mode domain, sliding mode dynamics, the existence of four types of equilibria and tangent point of Filippov system, regular/virtual equilibrium bifurcation, and boundary node bifurcations. In addition, the local stability of pseudoequilibrium implies global stability in our numerical simulations. Globally touching bifurcation especially indicates that the density of pest can be successfully maintained below the ET by designing suitable threshold policy strategy. Therefore our control objective can be achieved in the above two cases, which are desired situations in crop, livestock sectors and forestry.

The organization of this paper is as follows: in Section 2, we give some basic results and preliminaries for ODE system and Filippov system. In Section 3, the existence of sliding segments and sliding mode dynamics for the Filippov system (3) are addressed. In Section 4, we give the null-isoclines and equilibria. Based on those results, in Section 5, we consider the bifurcation sets of equilibria and sliding bifurcation analyses. Then, in the last section, we give some discussions.

\section{The ODE System and Filippov System}

2.1. The Basic Preliminaries and Results for ODE System. The ODE model (1) has been well studied in $[2,7]$, and a complete classification of the asymptotic behavior of the solutions of the ratio-dependent model with Michaelis-Menten functional response has been proposed. In the following, we present some primary results in the following Lemma which are useful for this study.

Lemma 1. System (1) includes three equilibria $(0,0),(k, 0)$, and a unique positive equilibrium $E^{*}\left(x^{*}, y^{*}\right)$ if and only if the following two conditions are true: $\beta a-\delta>0$ and $r>(\beta a-$ $\delta) / \beta b$, where $E^{*}=\left(x^{*}, y^{*}\right)=(k(\beta b r+\delta-\beta a) / \beta b r, k(\beta a-$ $\left.\delta)(\beta b r+\delta-\beta a) / \beta b^{2} r \delta\right)$. If $\beta a-\delta>0$ and $\beta a-\delta<r \leq$ $(a-b \delta) / b,(0,0)$ is globally asymptotically stable; if $\beta a-\delta<0$ and $r \geq(a-b \delta) / b,(k, 0)$ is globally asymptotically stable; if $\beta a-\delta>0$ and $(\beta a-\delta) / \beta b<r \leq \beta a-\delta$ or $\beta a-\delta>0$, $r>\beta a-\delta$, and $r \geq(a-b \delta) / b$, the positive equilibrium of $E^{*}=\left(x^{*}, y^{*}\right)$ is globally asymptotically stable. However if the positive equilibrium is locally asymptotically stable, then the system (1) has no nontrivial positive periodic solutions. If $\beta a-\delta>0$ and $\max \{\beta a-\delta,(\beta a-\delta) / \beta b\}<r<(a-b \delta) / b$ hold true, then the system (1) has at most one stable limit cycle.

2.2. Filippov Ratio-Dependent Prey-Predator Model and Preliminaries. By now, based on IPM strategies and TPC, the models (1) and (2) can be incorporated and rewritten in the following form:

$$
\begin{gathered}
\dot{x}(t)=r x\left(1-\frac{x}{k}\right)-\frac{a x y}{b y+x}-\varepsilon q_{1} x, \\
\dot{y}(t)=-\delta y+\frac{\beta a x y}{b y+x}+\varepsilon q_{2} y,
\end{gathered}
$$


with

$$
\varepsilon= \begin{cases}0, & x<\mathrm{ET} \\ 1, & x>\mathrm{ET} .\end{cases}
$$

We first introduce some useful properties and definitions on Filippov system according to [24, 25], so that we can investigate the model (3) in more detail. Let $H(Z)=x$ - ET with vector $Z=(x, y)^{T}$, and

$$
\begin{aligned}
& F_{S_{1}}(Z) \\
& \quad=\left(r x\left(1-\frac{x}{k}\right)-\frac{a x y}{b y+x},-\delta y+\frac{\beta a x y}{b y+x}\right)^{T}, \\
& F_{S_{2}}(Z) \\
& \quad=\left(r x\left(1-\frac{x}{k}\right)-\frac{a x y}{b y+x}-q_{1} x,-\delta y+\frac{\beta a x y}{b y+x}+q_{2} y\right)^{T} .
\end{aligned}
$$

Then the system (3) can be rewritten as the following Filippov system:

$$
\dot{Z}(t)= \begin{cases}F_{S_{1}}(Z), & Z \in S_{1}, \\ F_{S_{2}}(Z), & Z \in S_{2} .\end{cases}
$$

In addition, we define the discontinuity boundary set (or the switching line) $\Sigma=\left\{Z \in R_{+}^{2} \mid H(Z)=0\right\}$, which divides $R_{+}^{2}$ into two regions $S_{1}$ and $S_{2}$, where

$$
\begin{aligned}
& S_{1}=\left\{Z \in R_{+}^{2} \mid H(Z)<0\right\}, \\
& S_{2}=\left\{Z \in R_{+}^{2} \mid H(Z)>0\right\} .
\end{aligned}
$$

From now on, Filippov system (3) in different regions $S_{1}$ or $S_{2}$ is named as system $S_{1}$ (i.e., system (1)) or system $S_{2}$ (i.e., system (2)) correspondingly.

Denote

$$
\sigma(Z)=\left\langle H_{Z}(Z), F_{S_{1}}(Z)\right\rangle\left\langle H_{Z}(Z), F_{S_{2}}(Z)\right\rangle,
$$

where $H_{Z}$ is a nonvanishing gradient of the smooth scale function $H$ on $\Sigma$, and $\langle\cdot\rangle$ denotes the standard scalar product; then the sliding mode domain can be defined as

$$
\Sigma_{S}=\{Z \in \Sigma \mid \sigma(Z) \leq 0\} .
$$

We distinguish the following regions on $\Sigma$ :

(i) $\Sigma_{1} \in \Sigma$ is the escaping region if $\left\langle H_{Z}(Z), F_{S_{1}}(Z)\right\rangle<0$ and $\left\langle H_{Z}(Z), F_{S_{2}}(Z)\right\rangle>0$ on $\Sigma_{1}$;

(ii) $\Sigma_{2} \in \Sigma$ is the sliding region if $\left\langle H_{Z}(Z), F_{S_{1}}(Z)\right\rangle>0$ and $\left\langle H_{Z}(Z), F_{S_{2}}(Z)\right\rangle<0$ on $\Sigma_{2}$;

(iii) $\Sigma_{3} \in \Sigma$ is the sewing region if $\left\langle H_{Z}(Z), F_{S_{1}}(Z)\right\rangle$ $\left\langle H_{Z}(Z), F_{S_{2}}(Z)\right\rangle>0$ on $\Sigma_{3}$.

The following definitions about all types of equilibria for Filippov system are necessary throughout the paper, so we list them as follows.
Definition 2. A point $Z^{*}$ is called a regular equilibrium of system (3) if $F_{S_{1}}\left(Z^{*}\right)=0$, with $H\left(Z^{*}\right)<0$, or $F_{S_{2}}\left(Z^{*}\right)=0$, with $H\left(Z^{*}\right)>0$. A point $Z^{*}$ is called a virtual equilibrium of system (3), if $F_{S_{1}}\left(Z^{*}\right)=0$, with $H\left(Z^{*}\right)>0$, or $F_{S_{2}}\left(Z^{*}\right)=0$, with $H\left(Z^{*}\right)<0$.

Definition 3. A point $Z^{*}$ is called a pseudoequilibrium if it is an equilibrium of the sliding mode of system (3); that is, $(1-\lambda) F_{S_{1}}\left(Z^{*}\right)+\lambda F_{S_{2}}\left(Z^{*}\right)=0, H\left(Z^{*}\right)=0$, and $0<\lambda<1$, where

$$
\lambda=\frac{\left\langle H_{Z}(Z), F_{S_{1}}(Z)\right\rangle}{\left\langle H_{Z}(Z), F_{S_{1}}(Z)-F_{S_{2}}(Z)\right\rangle} .
$$

Definition 4. A point $Z^{*}$ is called a boundary equilibrium of system (3) if $F_{S_{1}}\left(Z^{*}\right)=0$, with $H\left(Z^{*}\right)=0$, or $F_{S_{2}}\left(Z^{*}\right)=0$, with $H\left(Z^{*}\right)=0$.

Definition 5. A point $Z^{*}$ is called a tangent point of system (3) if $Z^{*} \in \Sigma_{S}$ and $\left\langle H_{Z}\left(Z^{*}\right), F_{S_{1}}\left(Z^{*}\right)\right\rangle=0$, or $\left\langle H_{Z}\left(Z^{*}\right), F_{S_{2}}\left(Z^{*}\right)\right\rangle=0$.

The details and knowledge about the Filippov system, such as the concepts of Filippov solution, sliding mode solution, and bifurcation can be found in reference [24].

\section{Sliding Region and Sliding Mode Dynamics}

A sliding mode exists if there are regions in the discontinuity boundary $\Sigma$, where the vectors for the two subsystems of the system (3) are directed towards each other. It is well known that two basic methods the so-called Filippov convex method [24] and Utkin equivalent control method [18], are developed for the sliding mode and its domains, which are shown in the appendix.

3.1. Sliding Segment and Region. Based on the appendix, we have

$$
\lambda(Z)=\frac{r(1-\mathrm{ET} / k)-a y /(b y+\mathrm{ET})}{q_{1}},
$$

because the sliding mode regions can be determined by solving the inequalities; that is, $\lambda(Z) \geq 0$ and $\lambda(Z) \leq 1$. In order to solve the above two inequalities with respect to $y$, we need to consider the following two algebraic equations:

$$
\begin{gathered}
r\left(1-\frac{\mathrm{ET}}{k}\right)-\frac{a y}{b y+\mathrm{ET}}=0, \\
r\left(1-\frac{\mathrm{ET}}{k}\right)-\frac{a y}{b y+\mathrm{ET}}-q_{1}=0 .
\end{gathered}
$$

Solving the above two algebraic equations with respect to $y$ yields two roots, denoted by

$$
y_{1}=\frac{r \mathrm{ET}(k-\mathrm{ET})}{a k-b r(k-\mathrm{ET})}, \quad y_{2}=\frac{\left(r(k-\mathrm{ET})-q_{1} k\right) \mathrm{ET}}{a k-b\left(r(k-\mathrm{ET})-q_{1} k\right)} .
$$


Based on the relations between $y_{1}$ and $y_{2}$, there exist two cases for the existence of sliding segments of Filippov system (3). By simply calculating and arranging, we have the following results.

(i) When $r<a k / b(k-E T)$, the sliding segment can be described as

$$
\Sigma_{S}^{1}=\left\{(x, y) \mid \max \left\{0, y_{2}\right\} \leq y \leq y_{1}, x=\mathrm{ET}\right\} .
$$

(ii) When $\max \left\{q_{1} k /(k-\mathrm{ET}), a k / b(k-\mathrm{ET})\right\}<r<$ $\min \left\{k\left(a+b q_{1}\right) / b(k-\mathrm{ET}), a\left(k+b q_{1}\right) / b(k-\mathrm{ET})\right\}$, the sliding segment can be described as

$$
\Sigma_{S}^{2}=\left\{(x, y) \mid 0 \leq y \leq y_{2}, x=\mathrm{ET}\right\} .
$$

3.2. Sliding Mode Dynamics. Filippov system (3) only has one piece of sliding segment, and the solutions defined in it can be obtained from the sliding mode dynamics, which can be determined by employing the Utkin equivalent control method (see the appendix).

From $H=0$, we get that

$$
H_{Z}=x^{\prime}=r x\left(1-\frac{x}{k}\right)-\frac{a x y}{b y+x}-\varepsilon q_{1} x=0, \quad x=\mathrm{ET} .
$$

And solving the above equations with respect to $\varepsilon$ yields

$$
\varepsilon=\frac{r(1-\mathrm{ET} / k)-a y /(b y+\mathrm{ET})}{q_{1}} .
$$

Hence, the dynamics on the sliding mode $\Sigma_{S}$ can be determined by the following scalar differential equation:

$$
\begin{aligned}
\dot{y}(t)= & -\delta y+\frac{\beta a y \mathrm{ET}}{b y+\mathrm{ET}} \\
& +\frac{r(1-\mathrm{ET} / k)-a y /(b y+\mathrm{ET})}{q_{1}} q_{2} y, \\
= & P_{1}(y)\left(P_{2} y+P_{3}\right) \triangleq \phi(y),
\end{aligned}
$$

where $y \in \Sigma_{S}^{1}$ or $\Sigma_{S}^{2}, P_{1}(y)=y / q_{1}(b y+\mathrm{ET}), P_{2}=-\delta b q_{1}+$ $r b q_{2}(1-\mathrm{ET} / k)-a q_{2}$, and $P_{3}=-\delta q_{1} \mathrm{ET}+\beta a q_{1} \mathrm{ET}+r q_{2}(1-$ $\mathrm{ET} / k) \mathrm{ET}$.

\section{Null-Isoclines and Equilibria}

4.1. The Null-Isoclines of Filippov System (3). Null-isoclines of both systems $S_{1}$ and $S_{2}$ are related to the existence of equilibria and are useful for analysis of sliding dynamic.

Null-isoclines $\dot{x}(t)=0$ and $\dot{y}(t)=0$ for both systems $S_{1}$ and $S_{2}$ can be determined as follows. For the system $S_{1}$, solving the equation of the null-isocline $\dot{x}(t)=0$, yeilds

$$
f_{S_{1}}(x)=\frac{r x(1-x / k)}{a-b r(1-x / k)},
$$

and null-isocline $\dot{y}(t)=0$ gives

$$
g_{S_{1}}(x)=\frac{(\beta a-\delta) x}{b \delta} .
$$

For the system $S_{2}$, solving the equation of the null-isocline $\dot{x}(t)=0$, yields

$$
f_{S_{2}}(x)=\frac{\left(r x(1-x / k)-q_{1}\right) x}{a+b q_{1}-b r(1-x / k)},
$$

and null-isocline $\dot{y}(t)=0$ gives

$$
g_{S_{2}}(x)=\frac{\left(\beta a-\left(\delta-q_{2}\right)\right) x}{b\left(\delta-q_{2}\right)} .
$$

4.2. The Equilibria of Filippov System (3). Because the solutions of Filippov system (3) are composed of connecting standard solutions in subsystems $S_{1}, S_{2}$ and sliding mode solutions on $\Sigma_{S}^{1}$ or $\Sigma_{S}^{2}$. The definitions of all types of equilibria of Filippov system have been provided in Section 2, which are important to bifurcation analysis. There may be several types of equilibria for Filippov system (3) which include regular equilibrium (denoted by $E_{R}$ ), virtual equilibrium (denoted by $E_{V}$ ), pseudoequilibrium (denoted by $E_{P}$ ), boundary equilibrium (denoted by $E_{B}$ ), and one type of special point named as tangent point (denoted by $E_{T}$ ). Detailed definitions of these equilibria and the tangent point can be found in the literature [24].

From Lemma 1, we know that the subsystem $S_{1}$ with $x<$ ET has three possible equilibria: $(0,0),(k, 0)$, and interior equilibrium,

$$
\begin{aligned}
E_{S_{1}}^{*} & =\left(x_{S_{1}}^{*}, y_{S_{1}}^{*}\right) \\
& =\left(\frac{k(\beta b r+\delta-\beta a)}{\beta b r}, \frac{k(\beta a-\delta)(\beta b r+\delta-\beta a)}{\beta b^{2} r \delta}\right) .
\end{aligned}
$$

If $\beta a-\delta>0$ and $r>(\beta a-\delta) / \beta b$, then there is a positive interior equilibrium for the system $S_{1}$.

Equilibria for the subsystem $S_{2}$ with $x>$ ET satisfy

$$
\begin{gathered}
r x\left(1-\frac{x}{k}\right)-\frac{a x y}{b y+x}-q_{1} x=0, \\
-\delta y+\frac{\beta a x y}{b y+x}+q_{2} y=0 .
\end{gathered}
$$

Solving the above equations with respect to $x$ and $y$ yields three possible equilibria, that is, $(0,0),\left(k\left(r-q_{1}\right) / r, 0\right)$, and interior equilibrium,

$$
\begin{aligned}
E_{S_{2}}^{*}= & \left(x_{S_{2}}^{*}, y_{S_{2}}^{*}\right) \\
= & \left(\frac{k\left(\beta b\left(r-q_{1}\right)+\delta-q_{2}-\beta a\right)}{\beta b r},\right. \\
& \left.\frac{k\left(\beta a-\delta+q_{2}\right)\left(\beta b\left(r-q_{1}\right)+\delta-q_{2}-\beta a\right)}{\beta b^{2} r\left(\delta-q_{2}\right)}\right),
\end{aligned}
$$


which indicates that, if $r>\left(\beta a-\delta+q_{2}\right) / \beta b+q_{1}$ and $q_{2}<$ $\delta<\beta a+q_{2}$, then there is a positive interior equilibrium for the system $S_{2}$.

4.2.1. Regular Equilibria. For the subsystem $S_{1}$ with $x<$ ET, $(0,0)$ is a regular equilibrium, while $(k, 0)$ is a virtual equilibrium. In addition, according to the coordinate of equilibrium $E_{S_{1}}^{*}$, we have the following results. If $r>(\beta a-\delta) / \beta b>0$ and

$$
\frac{k(\beta b r+\delta-\beta a)}{\beta b r}<\mathrm{ET},
$$

then it is a regular equilibrium for the system $S_{1}$, denoted by $E_{R}^{1}$. If $r>(\beta a-\delta) / \beta b>0$ and

$$
\frac{k(\beta b r+\delta-\beta a)}{\beta b r}>\mathrm{ET},
$$

then the equilibrium $E_{S_{1}}^{*}$ becomes a virtual equilibrium, denoted by $E_{V}^{1}$.

About regular equilibria for the subsystem $S_{2}$ with $x>$ ET, $(0,0)$ is a virtual equilibrium, while $\left(k\left(r-p_{1}\right) / r, 0\right)$ may be a regular or virtual equilibrium which depends on the parameter space. Moreover, according to the coordinate of equilibrium $E_{S_{2}}^{*}$, we have the following conclusions. If $r>$ $\left(\beta a-\delta+q_{2}\right) / \beta b+q_{1}, q_{2}<\delta<\beta a+q_{2}$ and

$$
\frac{k\left(\beta b\left(r-q_{1}\right)+\delta-q_{2}-\beta a\right)}{\beta b r}>\mathrm{ET},
$$

then it is a regular equilibrium for the system $S_{2}$, denoted by $E_{R}^{2}$. Note that if $r>\left(\beta a-\delta+q_{2}\right) / \beta b+q_{1}, q_{2}<\delta<\beta a+q_{2}$ and

$$
\frac{k\left(\beta b\left(r-q_{1}\right)+\delta-q_{2}-\beta a\right)}{\beta b r}<\mathrm{ET},
$$

then the equilibrium $E_{S_{2}}^{*}$ becomes a virtual equilibrium, denoted by $E_{V}^{2}$. Based on the above analysis, if

$$
\frac{k(\beta a-\delta)}{\beta b(k-\mathrm{ET})}<r<\frac{k\left(\beta a-\delta+q_{2}+\beta b q_{1}\right)}{\beta b(k-\mathrm{ET})},
$$

then the two virtual equilibria $E_{V}^{1}$ and $E_{V}^{2}$ can coexist.

4.2.2. Pseudoequilibrium. For the existence of pseudoequilibrium $E_{P}=\left(\mathrm{ET}, y_{P}\right), y_{P}$ component of the pseudoequilibrium of sliding flow satisfies the following equation:

$$
P_{1}(y)\left(P_{2} y+P_{3}\right)=0,
$$

where $y \in \Sigma_{S}^{1}$ or $\Sigma_{S}^{2}$. Solving the above equation with respect to $y$ yields two possible roots denoted by $y_{P_{1}}=0$ and

$$
y_{P_{2}}=\frac{\left(\delta q_{1}-\beta a q_{1}-r q_{2}(1-\mathrm{ET} / k)\right) \mathrm{ET}}{-\delta b q_{1}+r b q_{2}(1-\mathrm{ET} / k)-a q_{2}} .
$$

Further, if

$$
\frac{k q_{1}(\delta-\beta a)}{q_{2}(k-\mathrm{ET})}<r<\frac{k\left(a q_{2}+\delta b q_{1}\right)}{b q_{2}(k-\mathrm{ET})}
$$

holds true, then $y_{P_{2}}$ is a positive root. Note that if $E_{P_{1}}=$ $\left(\mathrm{ET}, y_{P_{1}}\right)$ or $E_{P_{2}}=\left(\mathrm{ET}, y_{P_{2}}\right)$ lies in the sliding region $\Sigma_{S}^{1}$ or $\Sigma_{S}^{2}$, then the model has pseudoequilibrium. To do this, we consider the following two cases.

Case 1 (sliding segment defined by $\Sigma_{S}^{1}$ ). If the inequality $r<$ $q_{1} k /(k-\mathrm{ET})$ holds true, then $E_{P_{1}}=\left(\mathrm{ET}, y_{P_{1}}\right) \in \Sigma_{S}^{1}$ is a pseudoequilibrium of the Filippov system (3). Note that $-\delta b q_{1}+$ $r b q_{2}(1-\mathrm{ET} / k)-a q_{2}<0$ (i.e., $\left.r<k\left(a q_{2}+\delta b q_{1}\right) / b q_{2}(k-\mathrm{ET})\right)$ is well defined in this case. Therefore if the inequalities

$$
\begin{aligned}
\max & \left\{\frac{k q_{1}(\delta-\beta a)}{q_{2}(k-\mathrm{ET})}, \frac{k(\beta a-\delta)}{\beta b(k-\mathrm{ET})}\right\}<r \\
& <\min \left\{\frac{k\left(\beta a-\delta+q_{2}+\beta b q_{1}\right)}{\beta b(k-\mathrm{ET})}, \frac{k\left(a q_{2}+\delta b q_{1}\right)}{b q_{2}(k-\mathrm{ET})}\right\}
\end{aligned}
$$

hold true, then $E_{P_{2}}=\left(\mathrm{ET}, y_{P_{2}}\right) \in \Sigma_{S}^{1}$ is a positive pseudoequilibrium of the Filippov system (3).

Case 2 (sliding segment defined by $\Sigma_{S}^{2}$ ). In this case, $E_{P_{1}}=$ (ET, $\left.y_{P_{1}}\right) \in \Sigma_{S}^{2}$ is a pseudoequilibrium of the Filippov system (3). If the inequalities

$$
\begin{aligned}
\max & \left\{\frac{k\left(\beta a-\delta+q_{2}+\beta b q_{1}\right)}{\beta b(k-\mathrm{ET})}, \frac{k q_{1}(\delta-\beta a)}{q_{2}(k-\mathrm{ET})}\right\} \leq r \\
& <\frac{k\left(a q_{2}+\delta b q_{1}\right)}{b q_{2}(k-\mathrm{ET})}
\end{aligned}
$$

hold true, $E_{P_{2}}=\left(\mathrm{ET}, y_{P_{2}}\right) \in \Sigma_{S}^{2}$ is a positive pseudoequilibrium of the Filippov system (3).

4.2.3. Boundary Equilibrium. The boundary equilibria of Filippov system (3) satisfy

$$
\begin{gathered}
r x\left(1-\frac{x}{k}\right)-\frac{a x y}{b y+x}-\varepsilon q_{1} x=0, \\
-\delta y+\frac{\beta a x y}{b y+x}+\varepsilon q_{2} y=0, \\
x=\mathrm{ET},
\end{gathered}
$$

with $\varepsilon=0$ or 1 , which indicate that, if

$$
\begin{gathered}
\frac{k(\beta b r+\delta-\beta a)}{\beta b r}=\mathrm{ET} \\
\text { or } \frac{k\left(\beta b\left(r-q_{1}\right)+\delta-q_{2}-\beta a\right)}{\beta b r}=\mathrm{ET},
\end{gathered}
$$

then we have the boundary equilibria

$$
\begin{gathered}
E_{B}^{1}=\left(\mathrm{ET}, \frac{(\beta a-\delta) \mathrm{ET}}{b \delta}\right), \\
E_{B}^{2}=\left(\mathrm{ET}, \frac{\left(\beta a-\delta+q_{2}\right) \mathrm{ET}}{b\left(\delta-q_{2}\right)}\right) .
\end{gathered}
$$


4.2.4. Tangent Point. According to the definition of tangent point, we can see that the tangent point $E_{T}=\left(\mathrm{ET}, y_{T}\right)$ on sliding segment $\Sigma_{S}$ satisfies

$$
\begin{gathered}
r x\left(1-\frac{x}{k}\right)-\frac{a x y}{b y+x}-\varepsilon q_{1} x=0, \\
x=\mathrm{ET} .
\end{gathered}
$$

Solving the above equations with respect to $x$ and $y$ yields two tangent points, including

$$
\begin{aligned}
E_{T}^{1} & =\left(\mathrm{ET}, \frac{r \mathrm{ET}(1-\mathrm{ET} / k)}{a-b r(1-\mathrm{ET} / k)}\right), \\
E_{T}^{2} & =\left(\frac{\mathrm{ET}\left(r(1-\mathrm{ET} / k)-q_{1}\right)}{a+q_{1} b-b r(1-\mathrm{ET} / k)}\right) .
\end{aligned}
$$

If we fix all parameters, then the relations among nullisoclines, regular/virtual equilibria, pseudoequilibrium, and sliding segment are provided in Figure 1. Note that virtual equilibria of both systems $S_{1}$ and $S_{2}$ imply the existence of pseudoequilibrium, and we will prove this general result later.

4.3. The Stability of Pseudoequilibrium. In the process of pest control, we should apply all kinds of control strategies so as to prevent multiple pest outbreaks or make sure that the total density of the pest stabilizes at a desired level of ET. If the unique positive equilibrium of system $S_{1}$ and system $S_{2}$ is virtual simultaneously, then the sliding flow has a unique pseudoequilibrium. In order to realize this goal, we can choose a set of parameters such that all the equilibria of subsystems $S_{1}$ and $S_{2}$ are virtual equilibria, and the pseudoequilibria are globally stable, which have been widely used in pest control [26-28]. For example, if we fixed all parameter values as those in Figure 2, then both virtual equilibria coexist. Therefore, we address the stability of pseudoequilibrium $E_{P_{2}}$ in the following which is important to control pest.

Theorem 6. Either the inequalities (34) or (35) hold true or the two virtual equilibria $E_{V}^{1}$ and $E_{V}^{2}$ can coexist; then Filippov system (3) contains a positive pseudoequilibrium $E_{P_{2}}$. Regardless of which cases would occur, the pseudoequilibrium $E_{P_{2}}$ is locally stable with respect to sliding mode domain.

Proof. According to the conditions of Theorem 6 we see that the inequalities (30) hold true, which implies that the inequalities (34) are true. Based on the discussions about the existence of pseudoequilibrium in Section 4.2, we have concluded that if inequalities (34) or (35) hold true, then the system (3) contains a positive pseudoequilibrium $E_{P_{2}}$. It follows from (18) that we have

$$
\begin{aligned}
& \left.\frac{d \phi(y)}{d y}\right|_{y=y_{P_{2}}} \\
& =\frac{\left(-\delta b^{2} k q_{1}+r b^{2} q_{2}(k-\mathrm{ET})-a b k q_{2}\right) y_{P_{2}}^{2}}{k q_{1}\left(b y_{P_{2}}+\mathrm{ET}\right)^{2}}
\end{aligned}
$$

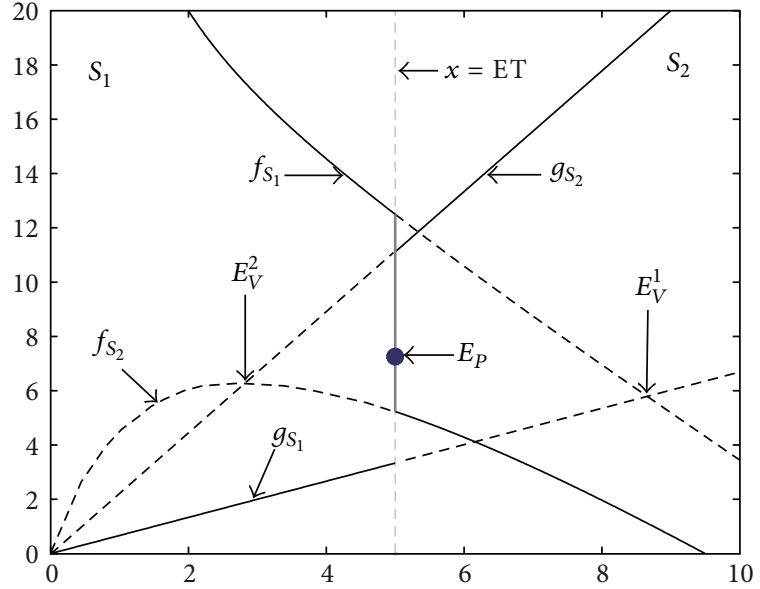

FIGURE 1: Notations for null-isoclines, regular/virtual equilibria, pseudoequilibrium, and sliding segment. The parameter values are fixed as follows: $a=0.7, b=0.6, r=1.2, k=12, \beta=0.5, \delta=0.25$, $q_{1}=0.25, q_{2}=0.1$, and $\mathrm{ET}=5$.

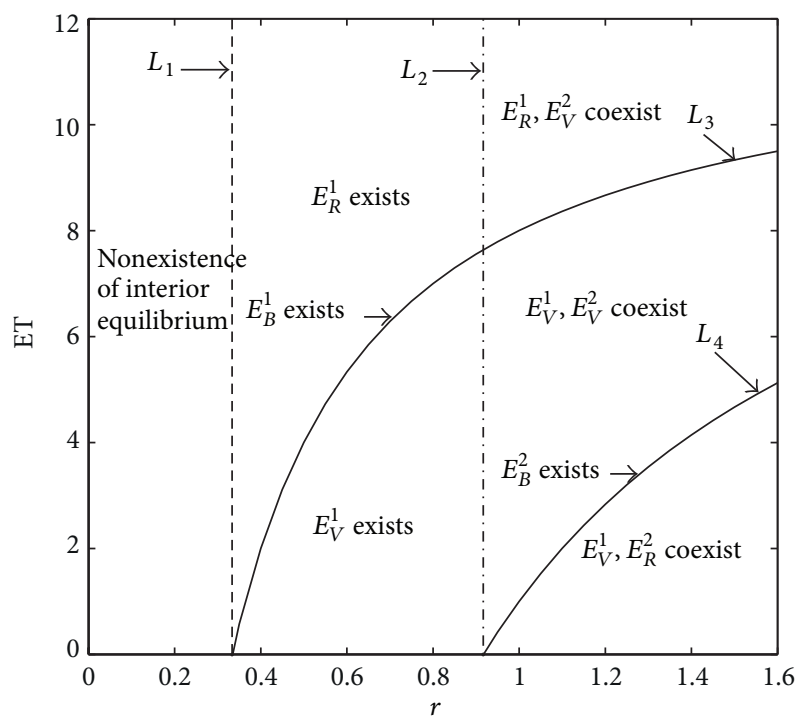

FIGURE 2: Regular/virtual equilibrium. The parameter values are fixed as follows: $a=0.7, b=0.6, k=12, \beta=0.5, \delta=0.25, q_{1}=0.25$, and $q_{2}=0.1$.

$$
\begin{aligned}
& +\frac{\left(-2 \delta b k q_{1} \mathrm{ET}-2 a k q_{2} \mathrm{ET}+2 r b q_{2} \mathrm{ET}(k-\mathrm{ET})\right) y_{P_{2}}}{k q_{1}\left(b y_{P_{2}}+\mathrm{ET}\right)^{2}} \\
& +\frac{-\delta k q_{1} \mathrm{ET}^{2}+a \beta k q_{1} \mathrm{ET}^{2}+r k q_{2} \mathrm{ET}^{2}-r q_{2} \mathrm{ET}^{3}}{k q_{1}\left(b y_{P_{2}}+\mathrm{ET}\right)^{2}} \\
& =\frac{\left(k q_{1}(\delta-\beta a)-r q_{2}(k-\mathrm{ET})\right)\left(\delta b k q_{1}-r b q_{2}(k-\mathrm{ET})+a k q_{2}\right)}{a q_{1} k^{2}\left(b \beta q_{1}+q_{2}\right)} .
\end{aligned}
$$

Therefore, if the inequalities (33) hold true, then $d \phi(y) /$ $d y<0$ and $y_{P_{2}}$ is a positive root of (31). Note that the inequalities (34) and (35) indicate the inequalities (33). Thus, the positive pseudoequilibrium $E_{P_{2}}$ is locally stale if it exists. 
It is difficult to directly prove the global stability of pseudoequilibrium $E_{P_{2}}$ in this case. Because we cannot employ the classical Bendixson-Dulac theorem due to the discontinuity of vector fields. However, if there is not crossing cycle surrounding sliding segment, then pseudoequilibrium is globally stable [29]. From the analysis of global bifurcation for the system (3) in the following section, the system just has touching bifurcation and there is nonexistence of a sliding cycle which surrounds the $E_{P_{2}}$. By using the similar methods, we have that pseudoequilibrium $E_{P_{2}}$ is globally stable. That is to say, the local stability of pseudoequilibrium $E_{P_{2}}$ with respect to sliding mode domain indicates its global stability in the first quadrant (shown in Figures 3(a), 3(b), 4(b), and 4(c)). In practice, in order to control pest outbreak, we should choose the desirable ET at first, so that all equilibria of each system such as system $S_{1}$ and system $S_{2}$ become virtual; then pseudoequilibrium not only exists but also is globally stable. In other words, the density of pest can be stable at the ET. When the density of pest reaches or exceeds the ET, we should carry out control strategies (e.g., releasing natural enemy, etc.), until it falls below the ET. In this way, our control goal can be realized fully.

\section{Equilibria and Sliding Bifurcation Set}

5.1. Regular/Virtual Equilibrium Bifurcation. According to the above discussions, it is obvious that $r$ and ET are primary factors in determining the existence of the above different types of equilibria of the system (3). So we define four curves about parameters $r$ and ET as follows:

$$
\begin{aligned}
& L_{1}=\left\{(r, \mathrm{ET}) \mid r=\frac{\beta a-\delta}{\beta b}\right\}, \\
& L_{2}=\left\{(r, \mathrm{ET}) \mid r=\frac{\beta a-\delta+q_{2}}{\beta b}+q_{1}\right\}, \\
& L_{3}=\left\{(r, \mathrm{ET}) \mid \mathrm{ET}=\frac{k(\beta b r+\delta-\beta a)}{\beta b r}\right\}, \\
& L_{4}=\left\{(r, \mathrm{ET}) \mid \mathrm{ET}=\frac{k\left(\beta b\left(r-q_{1}\right)+\delta-q_{2}-\beta a\right)}{\beta b r}\right\} .
\end{aligned}
$$

The four curves (i.e., $L_{1}, L_{2}, L_{3}$, and $L_{4}$ ) divide the $r$ and ET parameter space into six regions, and the existence or coexistence of regular or virtual equilibria is indicated in each region. The boundary equilibria $E_{B}^{1}$ and $E_{B}^{2}$ can appear on the lines $L_{3}$ and $L_{4}$ accordingly. In particular, it follows from Figure 2 that the two virtual equilibria $E_{V}^{1}$ and $E_{V}^{2}$ can coexist, which is very important to pest control. However, the two regular equilibria $E_{R}^{1}$ and $E_{R}^{2}$ cannot coexist.

5.2. Boundary Node Bifurcations. This type of bifurcation may occur for Filippov system (3) once equilibria $E_{P}, E_{T}$, and $E_{R}$ or $E_{T}$ and $E_{R}$ collide together simultaneously when ET passes through a critical value. In this part, we choose ET as bifurcation parameter, and all other parameters are fixed as those in Figure 3. Note that once the parameter ET passes through the first critical value $\mathrm{ET}_{c_{1}}=9.3333$, the regular equilibrium $E_{R}^{1}$, tangent point $E_{T}$, and pseudoequilibrium $E_{P}$ collide together (see Figure 3(b)), where $\mathrm{ET}_{c_{1}}$ is determined by

$$
\mathrm{ET}_{c_{1}}=\frac{k(\beta b r+\delta-\beta a)}{\beta b r} .
$$

A virtual equilibrium $E_{V}^{1}$, an invisible tangent point $E_{T}^{1}$ and a pseudoequilibrium $E_{P}$ coexist, as shown in Figure 3(a), when $\mathrm{ET}<\mathrm{ET}_{c_{1}}$. They collide at $\mathrm{ET}=\mathrm{ET}_{c_{1}}$ and are substituted by a visible tangent point $E_{T}^{1}$, as shown in Figure 3(c), when $\mathrm{ET}>\mathrm{ET}_{c_{1}}$.

Similarly, another boundary node bifurcation of Filippov system (3) occurs at $\mathrm{ET}_{\mathcal{c}_{2}}=4.6667$ (see Figure 4(b)), where $\mathrm{ET}_{c_{2}}$ is determined by

$$
\mathrm{ET}_{\mathcal{C}_{2}}=\frac{k\left(\beta b\left(r-q_{1}\right)+\delta-q_{2}-\beta a\right)}{\beta b r} .
$$

A stable regular equilibrium $E_{R}^{2}$ and a visible tangent point $E_{T}^{2}$ coexist as shown in Figure $4(\mathrm{a})$ when $\mathrm{ET}<\mathrm{ET}_{\mathcal{c}_{2}}$. They collide with a pseudoequilibrium $E_{P}$ at $\mathrm{ET}=\mathrm{ET}_{c_{2}}$ and are substituted by an invisible tangent point $E_{T}^{2}$ as shown in Figure 4(c) when $\mathrm{ET}>\mathrm{ET}_{c_{2}}$.

5.3. Global Sliding Bifurcation. Global sliding bifurcations involve nonvanishing cycles, which include sliding disconnection, touching (or grazing) bifurcation, buckling bifurcation, crossing bifurcation, bifurcation of a sliding homoclinic orbit, and heteroclinic orbit [22]. Touching (or grazing) bifurcation implies that a positive period solution can collide with the sliding segments. From the work of Kuang and Beretta and Hsu et al. [2,7], they concluded that when the value of the parameter $a$ passed slightly through the bifurcation value, a stable limit cycle bifurcates from the unstable positive interior equilibrium for the system (1). According to numerical simulations, the system (3) just has touching bifurcation. For example, if we choose ET as bifurcation parameter and fix all other parameters as shown in Figure 5, when the value of parameter ET varies, touching bifurcation occurs at the critical value $\mathrm{ET}_{c_{3}}=2.59$ for the system (3). Note that extensive numerical simulations indicate that, no matter what the value of ET is in touching bifurcation, the whole periodic solution lies in the region $S_{1}$ (shown in Figure 5). This implies that the density of pest can be successfully maintained below ET by designing suitable threshold policy strategies. So our control objective can be fully realized, which is a desired situation in crop, livestock sectors and forestry.

\section{Discussion}

Recently, the threshold policy and IPM strategies have attracted great attention in agriculture, forestry, animal husbandry, and so on [10-12]. In the process of pest control, IPM strategies would be used only when the density of pest reaches or exceeds the ET. In addition, Filippov system provides a natural and rational framework for those real world problems, so it has been widely used in different fields 


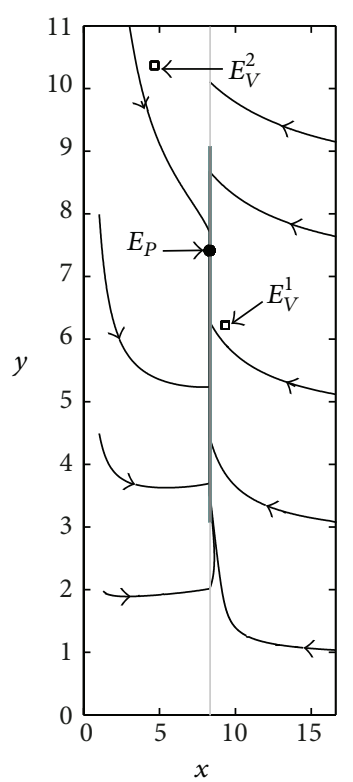

(a) $\mathrm{ET}=8.3$

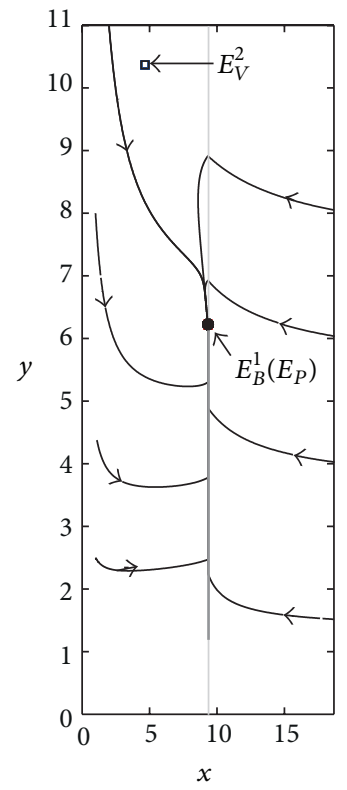

(b) $\mathrm{ET}=9.3333$

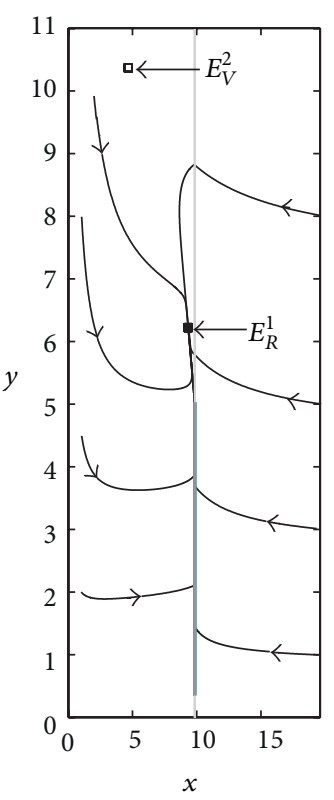

(c) $\mathrm{ET}=9.8$

FIGURE 3: Boundary node bifurcation for Filippov system (3). The parameter values are fixed as follows: $a=0.7, b=0.6, r=1.5, k=12$, $\beta=0.5, \delta=0.25, q_{1}=0.25$, and $q_{2}=0.1$.

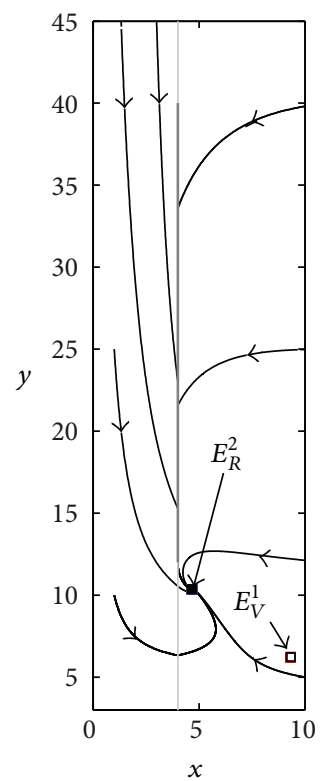

(a) $\mathrm{ET}=4$

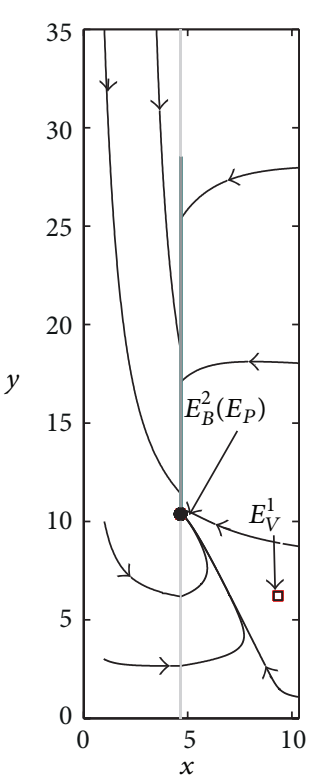

(b) $\mathrm{ET}=4.6667$

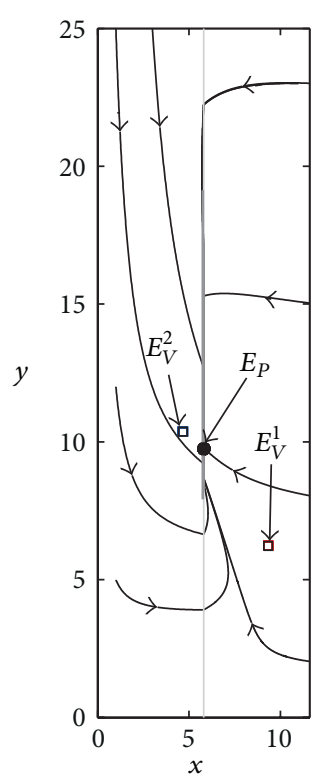

(c) $\mathrm{ET}=5.8$

Figure 4: Boundary node bifurcation for Filippov system (3). The parameter values are fixed as follows: $a=0.7, b=0.6, r=1.5, k=12$, $\beta=0.5, \delta=0.25, q_{1}=0.25$, and $q_{2}=0.1$.

such as in science and engineering [19-21, 23]. In this paper, we employ the sliding analysis of Filippov system to describe and investigate the long term dynamical behavior of the ratio-dependent-type prey-predator model with MichaelisMenten-type functional response, so that we can use the Filippov system to model intervention of pest control policy. Firstly, we investigate the sliding mode domain, sliding mode dynamics. Secondly, the null-isoclines and the existence of four types of equilibria for Filippov system, including regular, virtual, boundary, and pseudoequilibrium and the tangent points are discussed in detail. Moreover, the stability of pseudoequilibrium is also studied. Thirdly, we have investigated the local sliding bifurcations including regular/virtual equilibrium bifurcation, and boundary node bifurcations. Further, global touching bifurcation is also studied by numerical techniques. 


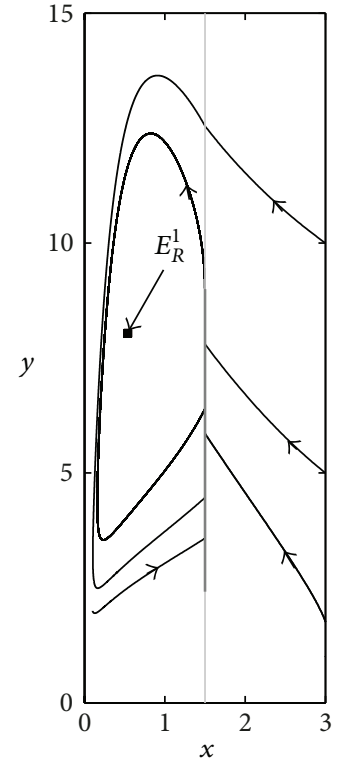

(a) $\mathrm{ET}=1.5$

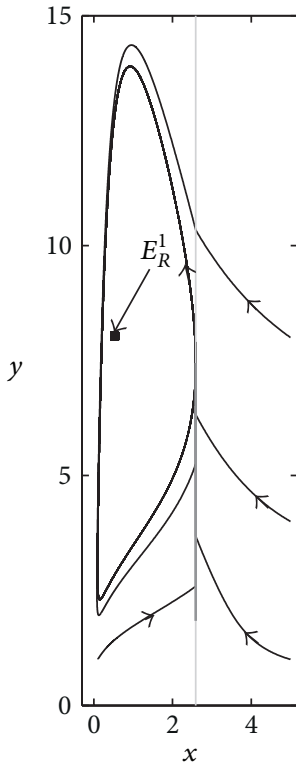

(b) $\mathrm{ET}=2.59$

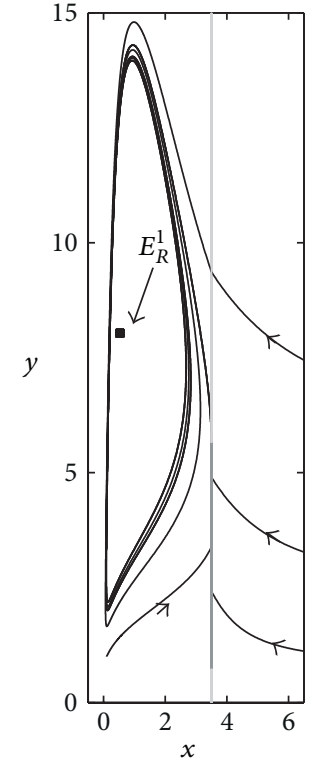

(c) $\mathrm{ET}=3.5$

FIGURE 5: Globally touching bifurcation for Filippov system (3). The parameter values are fixed as follows: $a=0.51, b=0.4, r=1.2, k=6$, $\beta=0.6863, \delta=0.05, q_{1}=0.4$, and $q_{2}=0.1$.

In the process of pest control, we should apply all kinds of control strategies so as to prevent multiple pest outbreaks or make sure that the density of pest stabilizes at a desired level of ET. In order to realize these goals, on the one hand, we can determine a set of parameters such that not only all the equilibria of subsystems $S_{1}$ and $S_{2}$ are virtual equilibria, which ensures that the pseudoequilibria exist, but also the pseudoequilibrium is globally stable. From Theorem 6 , we provided the conditions of the existence of pseudoequilibrium $E_{P_{2}}$ for the system (3) and showed that it is locally stable if it exists. Further, we showed that the local stability of pseudoequilibrium $E_{P_{2}}$ implies global stability by numerical simulation. On the other hand, globally touching bifurcation indicates that the density of pest can be successfully maintained below the ET by designing suitable threshold policy strategies (shown in Figure 5). Therefore our control objective can be achieved fully in the above two cases, which can be used to pest control in crop, livestock sectors and forestry.

Although impulsive prey-predator models with the ratiodependent- or Michaelis-Menten-type response function have been studied in [13-16], the Filippov systems have many advantages in describing interventions including spraying pesticides and releasing natural enemies compared with impulsive models. In this work, the number of natural enemy to be released is proportional to its number. It is interesting that releasing number of natural enemy can be described by constant, independently of the existing numbers of pest and natural enemy. Moreover, in practice, considering environmental energy resource finiteness, we should choose the total number of both populations as a guide to switch the system, which is called the weighted escapement policy (WEP) [19, $30-32]$. Therefore, in the future work, we will focus on the above two cases, which could result in richer dynamics.

\section{Appendix}

\section{Methods for Analysis of the Sliding Solution}

Filippov Convex Method. The Filippov method associates the following convex combination $F_{S}(Z)$ of the two vectors $F_{S_{1}}(Z)$ and $F_{S_{2}}(Z)$ with each nonsingular sliding point $Z \in$ $\Sigma_{2}$; that is,

$$
F_{S}(Z)=(1-\lambda(Z)) F_{S_{1}}(Z)+\lambda(Z) F_{S_{2}}(Z),
$$

where

$$
\lambda(Z)=\frac{\left\langle H_{Z}, F_{S_{1}}\right\rangle}{\left\langle H_{Z}, F_{S_{1}}-F_{S_{2}}\right\rangle},
$$

$F_{S}(Z)$ is tangent to $\Sigma_{2}$ and $0 \leq \lambda(Z) \leq 1$.

Thus, the sliding mode dynamics can be determined by

$$
\dot{Z}=F_{S}(Z), \quad Z \in \Sigma_{2},
$$

which is smooth on a one-dimensional sliding interval of $\Sigma_{2}$. The solution of the above equation is the sliding solution.

The equation $\lambda(Z)=0($ or $\lambda(Z)=1)$ indicates that the flow is determined by $F_{S_{1}}\left(\right.$ or $\left.F_{S_{2}}\right)$ alone. Therefore the sliding mode domain can be defined as

$$
\Sigma_{S}=\{Z=(x, y) \in \Sigma \mid 0 \leq \lambda(Z) \leq 1\},
$$

which is equivalent to $\Sigma_{S}=\{Z \in \Sigma \mid \sigma(Z) \leq 0\}$. Denote

$$
\Sigma_{S}^{+}=\{Z \in \Sigma \mid \lambda(Z)=1\}, \quad \Sigma_{S}^{-}=\{Z \in \Sigma \mid \lambda(Z)=0\},
$$


by the boundary of the sliding mode domain, so the vector fields are tangent to boundary $\Sigma_{S}^{+}$or $\Sigma_{S}^{-}$.

Utkin Equivalent Control Method. It follows from Utkin's works [18] on sliding mode dynamics along the manifold $\Sigma$; we note that Filippov system (3) can be also rewritten as follows:

$$
\dot{Z}=F\left(Z, U_{H}\right)
$$

where the control $U_{H}$ is defined as

$$
U_{H}= \begin{cases}0, & H(Z)<0, \\ U_{1}(Z, t), & H(Z)>0,\end{cases}
$$

where $U_{1}$ is a continuous function. In the controlled system (i.e., system $S_{2}$ ), the control $U_{H}=U_{1}$ is applied, and in the free system (i.e., system $S_{1}$ ) $U_{H}=0$ is applied. Assume that a sliding mode exists on manifold $\Sigma$; that is, $\Sigma_{2}$ is nonempty. Solving the following algebraic equation:

$$
\dot{H}=\frac{\partial H}{\partial Z} F\left(Z, U_{H}\right)=0
$$

with respect to $U_{H}$ on this manifold, gives the solution denoted by $U^{*}(Z, t)$, which is referred to as equivalent control. Substituting for $U_{H}$ in system (A.6) yields

$$
\dot{Z}=f\left(Z, U^{*}(Z, t)\right), \quad Z \in \Sigma_{2}
$$

which determines the sliding mode dynamics of the Filippov system (3).

\section{Acknowledgments}

This work is supported by the National Natural Science Foundation of China (NSFC, 11171199) and by the Fundamental Research Funds for the Central Universities (GK201003001).

\section{References}

[1] R. M. May, "Limit cycles in predator-prey communities," Science, vol. 177, no. 4052, pp. 900-902, 1972.

[2] Y. Kuang and E. Beretta, "Global qualitative analysis of a ratiodependent predator-prey system," Journal of Mathematical Biology, vol. 36, no. 4, pp. 389-406, 1998.

[3] W. Murdoch, C. Briggs, and R. Nisbet, Consumer-Resource Dynamics, Princeton University Press, New York, NY, USA, 2003.

[4] H. I. Freedman, Deterministic Mathematical Models in Population Ecology, vol. 57 of Monographs and Textbooks in Pure and Applied Mathematics, Marcel Dekker, New York, NY, USA, 1980.

[5] G. T. Skalski and J. F. Gilliam, "Functional responses with predator interference: viable alternatives to the Holling type II model," Ecology, vol. 82, no. 11, pp. 3083-3092, 2001.

[6] R. Arditi and L. R. Ginzburg, "Coupling in predator-prey dynamics: ratio-dependence," Journal of Theoretical Biology, vol. 139, no. 3, pp. 311-326, 1989.

[7] S.-B. Hsu, T.-W. Hwang, and Y. Kuang, "Global analysis of the Michaelis-Menten-type ratio-dependent predator-prey system," Journal of Mathematical Biology, vol. 42, no. 6, pp. 489506, 2001.
[8] C. Jost, O. Arino, and R. Arditi, "About deterministic extinction in ratio-dependent predator-prey models," Bulletin of Mathematical Biology, vol. 61, no. 1, pp. 19-32, 1999.

[9] D. Xiao and S. Ruan, "Global dynamics of a ratio-dependent predator-prey system," Journal of Mathematical Biology, vol. 43, no. 3, pp. 268-290, 2001.

[10] Z. Lu, X. Chi, and L. Chen, "Impulsive control strategies in biological control of pesticide," Theoretical Population Biology, vol. 64, no. 1, pp. 39-47, 2003.

[11] S. Tang and R. A. Cheke, "Models for integrated pest control and their biological implications," Mathematical Biosciences, vol. 215, no. 1, pp. 115-125, 2008.

[12] J. C. Van Lenteren and J. Woets, "Biological and integrated pest control in greenhouses," Annual Review of Entomology, vol. 33, pp. 239-250, 1988.

[13] B. Dai, H. Su, and D. Hu, "Periodic solution of a delayed ratiodependent predator-prey model with monotonic functional response and impulse," Nonlinear Analysis: Theory, Methods \& Applications, vol. 70, no. 1, pp. 126-134, 2009.

[14] X. Liu, G. Li, and G. Luo, "Positive periodic solution for a twospecies ratio-dependent predator-prey system with time delay and impulse," Journal of Mathematical Analysis and Applications, vol. 325, no. 1, pp. 715-723, 2007.

[15] G. Jiang, Q. Lu, and L. Qian, "Complex dynamics of a Holling type II prey-predator system with state feedback control," Chaos, Solitons \& Fractals, vol. 31, no. 2, pp. 448-461, 2007.

[16] H. Baek and Y. Lim, "Dynamics of an impulsively controlled Michaelis-Menten type predator-prey system," Communications in Nonlinear Science and Numerical Simulation, vol. 16, no. 4, pp. 2041-2053, 2011.

[17] T. Zhao, Y. Xiao, and R. J. Smith, "Non-smooth plant disease models with economic thresholds," Mathematical Biosciences, vol. 241, no. 1, pp. 34-48, 2013.

[18] V. I. Utkin, Sliding Modes in Control and Optimization, Communications and Control Engineering Series, Springer, Berlin, Germany, 1992.

[19] M. I. S. Costa, E. Kaszkurewicz, A. Bhaya, and L. Hsu, "Achieving global convergence to an equilibrium population in predator-prey systems by the use of a discontinuous harvesting policy," Ecological Modelling, vol. 128, no. 2-3, pp. 89-99, 2000.

[20] B. L. Van De Vrande, D. H. Van Campen, and A. De Kraker, "Approximate analysis of dry-friction-induced stick-slip vibrations by a smoothing procedure," Nonlinear Dynamics, vol. 19, no. 2, pp. 157-169, 1999.

[21] S. H. Doole and S. J. Hogan, "A piecewise linear suspension bridge model: nonlinear dynamics and orbit continuation," Dynamics and Stability of Systems, vol. 11, no. 1, pp. 19-47, 1996.

[22] Yu. A. Kuznetsov, S. Rinaldi, and A. Gragnani, "One-parameter bifurcations in planar Filippov systems," International Journal of Bifurcation and Chaos in Applied Sciences and Engineering, vol. 13, no. 8, pp. 2157-2188, 2003.

[23] M. di Bernardo, C. J. Budd, A. R. Champneys et al., "Bifurcations in nonsmooth dynamical systems," SIAM Review, vol. 50, no. 4, pp. 629-701, 2008.

[24] A. F. Filippov, Differential Equations with Discontinuous Righthand Sides, vol. 18 of Mathematics and Its Applications (Soviet Series), Kluwer Academic, Dordrecht, The Netherlands, 1988.

[25] S. Tang, J. Liang, Y. Xiao, and R. A. Cheke, "Sliding bifurcations of Filippov two stage pest control models with economic thresholds," SIAM Journal on Applied Mathematics, vol. 72, no. 4, pp. 1061-1080, 2012. 
[26] M. I. da Silveira Costa and M. E. M. Meza, "Application of a threshold policy in the management of multispecies fisheries and predator culling," Mathematical Medicine and Biology, vol. 23, no. 1, pp. 63-75, 2006.

[27] M. E. Mendoza Meza, A. Bhaya, E. Kaszkurewicz, and M. I. Da Silveira Costa, "Threshold policies control for predator-prey systems using a control Liapunov function approach," Theoretical Population Biology, vol. 67, no. 4, pp. 273-284, 2005.

[28] F. Dercole, A. Gragnani, and S. Rinaldi, "Bifurcation analysis of piecewise smooth ecological models," Theoretical Population Biology, vol. 72, no. 2, pp. 197-213, 2007.

[29] W. Wang, "Backward bifurcation of an epidemic model with treatment," Mathematical Biosciences, vol. 201, no. 1-2, pp. 5871, 2006.

[30] I. Noy-Meir, "Stability of grazing systems: an application of predator-prey graphs," The Journal of Animal Ecology, vol. 63, no. 2, pp. 459-481, 1975.

[31] R. M. Colombo and V. Krrivan, "Selective strategies in food webs," Mathematical Medicine and Biology, vol. 10, no. 4, pp. 281-291, 1993.

[32] V. Křivan, "Optimal foraging and predator-prey dynamics," Theoretical Population Biology, vol. 49, no. 3, pp. 265-290, 1996. 


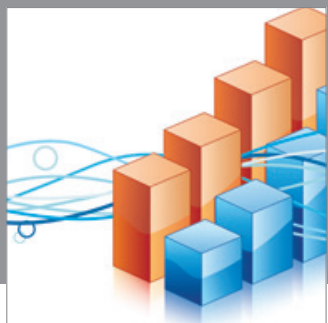

Advances in

Operations Research

mansans

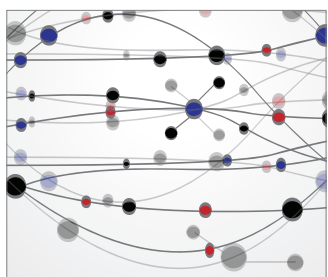

The Scientific World Journal
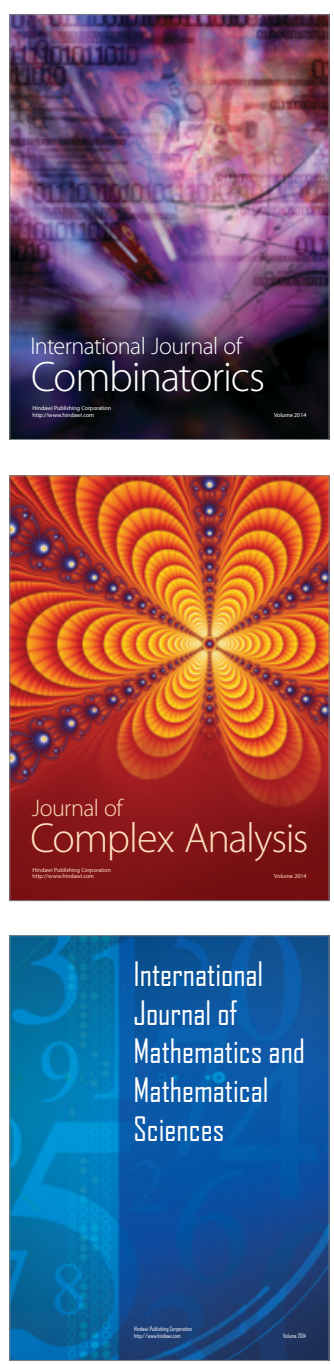
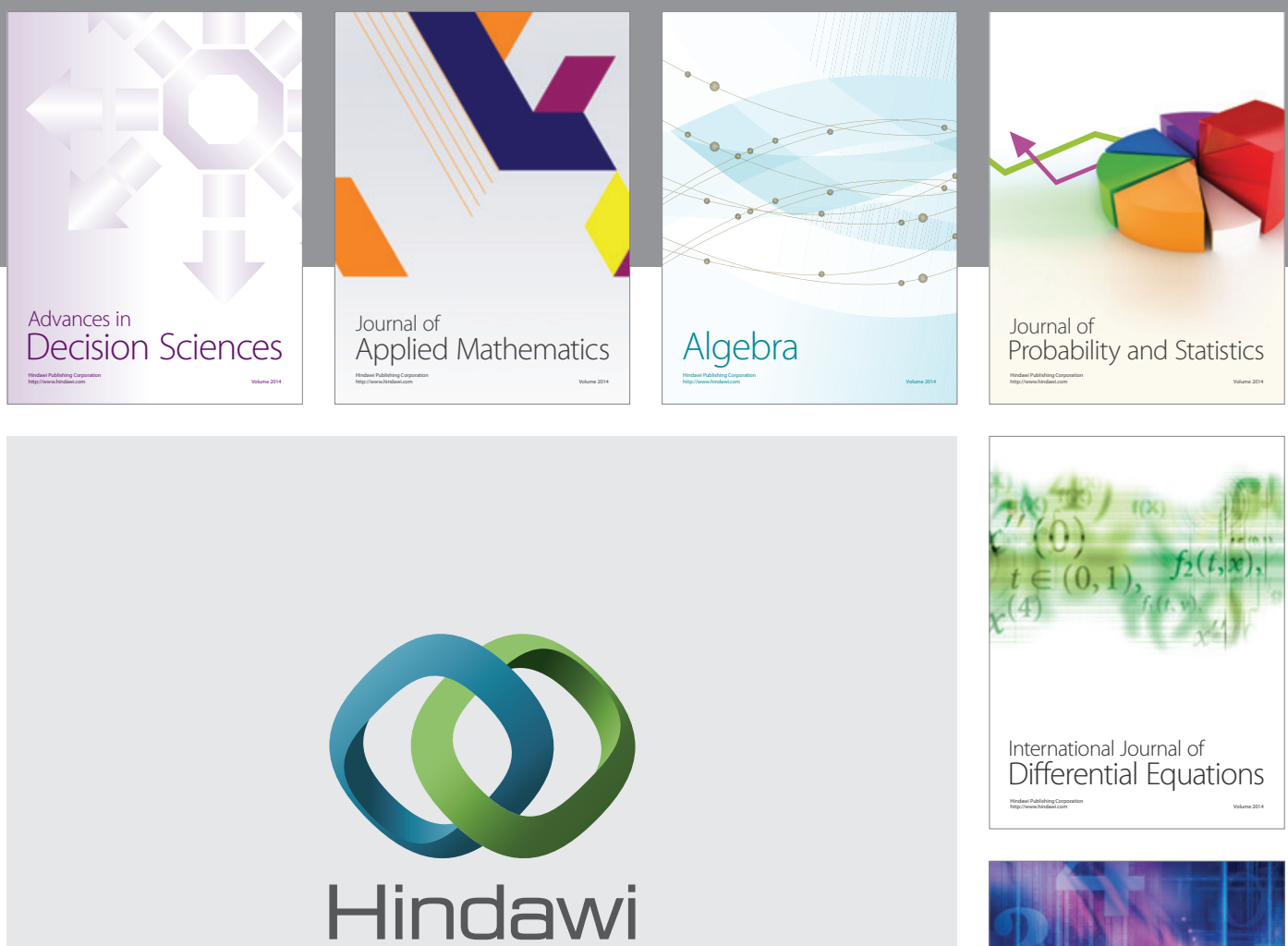

Submit your manuscripts at http://www.hindawi.com
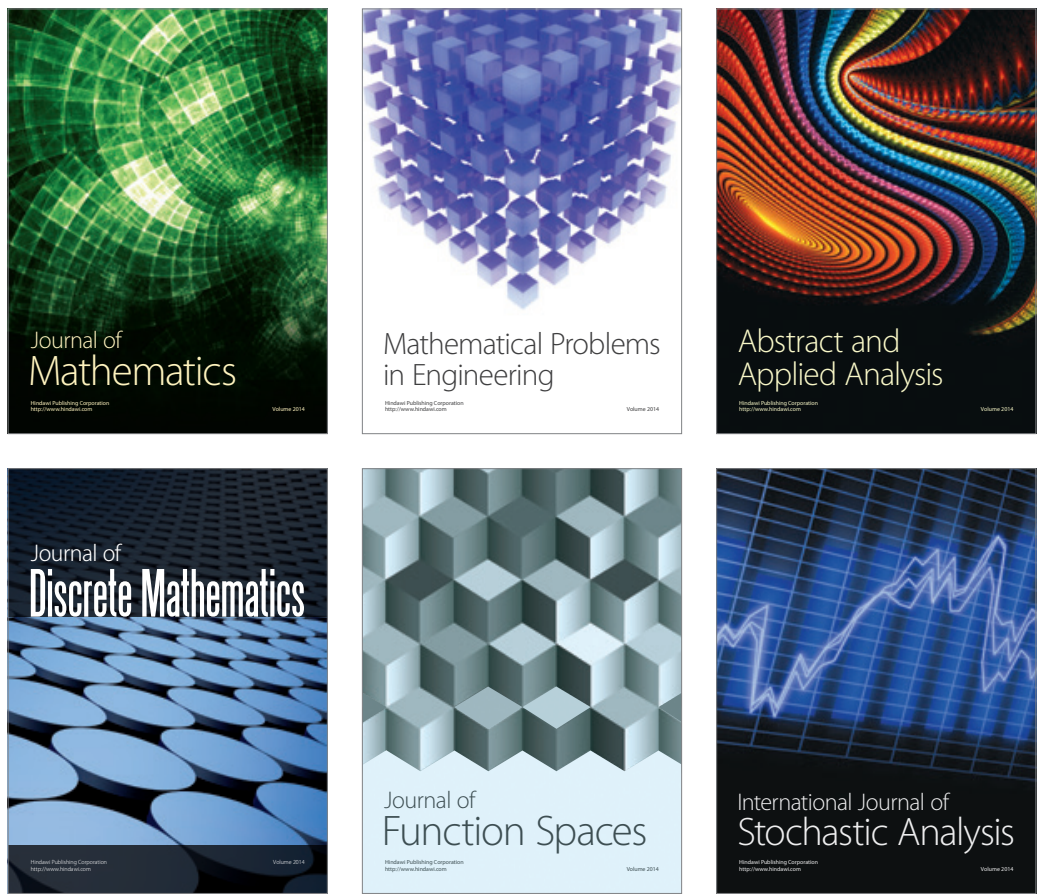

Journal of

Function Spaces

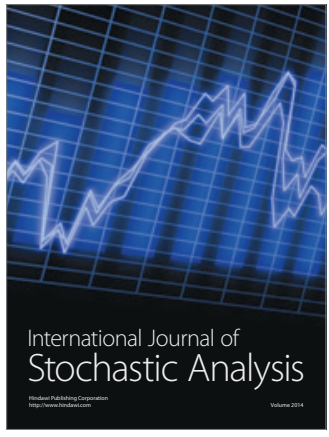

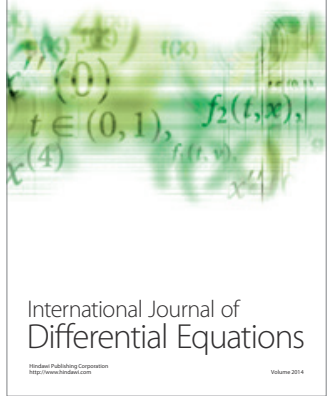
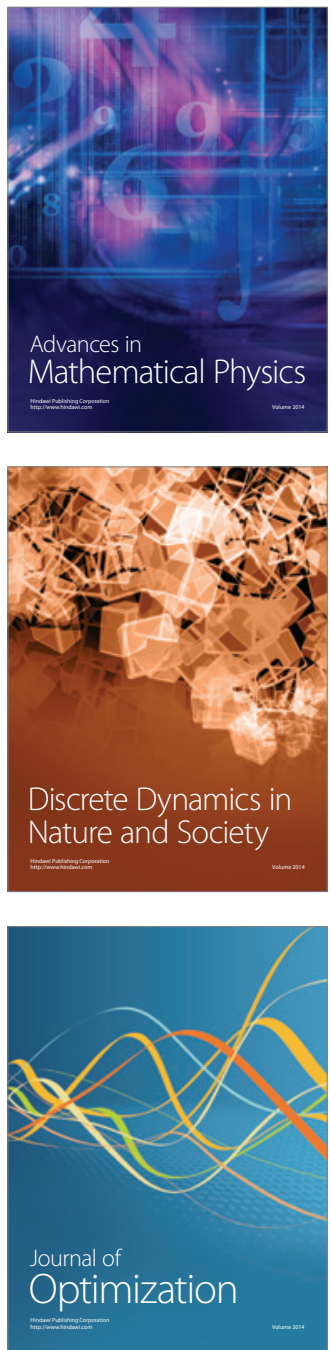